\title{
On Complete Spacelike Hypersurfaces in a Semi-Riemannian Warped Product
}

\author{
Yaning Wang ${ }^{1}$ and Ximin Liu ${ }^{2}$ \\ ${ }^{1}$ Department of Mathematics, South China University of Technology, Guangzhou, Guangdong 510641, China \\ ${ }^{2}$ School of Mathematical Sciences, Dalian University of Technology, Dalian, Liaoning 116024, China \\ Correspondence should be addressed to Yaning Wang; wyn051@163.com
}

Received 19 November 2012; Revised 26 January 2013; Accepted 26 January 2013

Academic Editor: P. G. L. Leach

Copyright (c) 2013 Y. Wang and X. Liu. This is an open access article distributed under the Creative Commons Attribution License, which permits unrestricted use, distribution, and reproduction in any medium, provided the original work is properly cited.

By applying Omori-Yau maximal principal theory and supposing an appropriate restriction on the norm of gradient of height function, we obtain some new Bernstein-type theorems for complete spacelike hypersurfaces with nonpositive constant mean curvature immersed in a semi-Riemannian warped product. Furthermore, some applications of our main theorems for entire vertical graphs in Robertson-Walker spacetime and for hypersurfaces in hyperbolic space are given.

\section{Introduction}

The theory of spacelike hypersurfaces immersed in semiRiemannian warped products with constant mean curvature has got increasing interest both from geometers and physicists recently. One of the basic questions on this topic is the problem of uniqueness for this type of hypersurfaces. The aim of this paper is to study such type problems. Before giving details of our main results, we firstly present a brief outline of some recent papers containing theorems related to ours.

By using a suitable application of well-known generalized maximal principal of Omori [1] and Yau [2], Albujer et al. [3] obtained uniqueness results concerning complete spacelike hypersurfaces with constant mean curvature immersed in a Robertson-Walker spacetime.

By applying a maximal principal due to Akutagawa [4], Aquino and de Lima [5] obtained a new Bernstein-type theorem concerning constant mean curvature complete vertical graphs immersed in a Riemannian warped product, which is supposed to satisfy an appropriate convergence condition defined as the following:

$$
0 \geq k_{M} \geq \sup _{I}\left(f^{\prime 2}-f f^{\prime \prime}\right),
$$

where $k_{M}$ denotes the sectional curvature of the fiber $M^{n}$, which is very different from null convergence condition (defined by (2)).
Replacing the null convergence condition by $(\ln f)^{\prime \prime} \leq 0$, Alías et al. obtained uniqueness theorems (see Section 2 of [6]) concerning compact spacelike hypersurface with higher constant mean curvature immersed in a spatially closed generalized Robertson-Walker spacetime. We also refer the reader to [7] for the other relevant results.

In this paper, following $[6,8]$, we consider the Laplacian of integral of warping function. By using a suitable maximal principal of Omori and Yau and supposing an appropriate restriction on the norm of gradient of height function, we obtain some new Bernstein-type theorems as the following.

Theorem 1. Let $\bar{M}^{n+1}=-I \times{ }_{f} M^{n}$ be a Robertson-Walker spacetime whose Riemannian fiber $M^{n}$ has constant sectional curvature $k$ satisfying the null convergence condition defined as the following:

$$
k \geq \sup _{I}\left(f f^{\prime \prime}-f^{\prime 2}\right) .
$$

Let $\psi: \Sigma^{n} \rightarrow \bar{M}^{n+1}$ be a complete spacelike hypersurface with constant mean curvature $H$. Suppose that $\Sigma^{n}$ is bounded away from the infinity of $\bar{M}^{n+1}$ and that

$$
H \leq \min \left\{\inf _{p \in \Sigma^{n}} \frac{f^{\prime}}{f}(h(p)), 0\right\} .
$$


If the height function $h$ of $\Sigma^{n}$ satisfies

$$
|\nabla h|^{2} \leq \alpha\left|\inf _{p \in \Sigma^{n}} \frac{f^{\prime}}{f}(h(p))-H\right|^{\beta},
$$

for some positive constant $\alpha$ and $\beta$, then $\Sigma^{n}$ is a slice.

Theorem 2. Let $\bar{M}^{n+1}=I \times_{f} M^{n}$ be a Riemannian warped product whose fiber $M^{n}$ has constant sectional curvature $k$ satisfying

$$
k \geq \sup _{I}\left(f^{\prime 2}-f f^{\prime \prime}\right) .
$$

Let $\psi: \Sigma^{n} \rightarrow \bar{M}^{n+1}$ be a complete spacelike hypersurface with constant mean curvature $\mathrm{H}$ and $\mathrm{H}_{2}$ is bounded from below. Suppose that $\Sigma^{n}$ is bounded away from the infinity of $\bar{M}^{n+1}$ and that

$$
\sup _{p \in \Sigma^{n}} \frac{f^{\prime}}{f}(h(p)) \leq H \leq 0 .
$$

If the height function $h$ of $\sum^{n}$ satisfies

$$
|\nabla h|^{2} \leq \alpha\left|H-\sup _{p \in \Sigma^{n}} \frac{f^{\prime}}{f}(h(p))\right|^{\beta},
$$

for some positive constant $\alpha$ and $\beta$, then $\Sigma^{n}$ is a slice.

\section{Preliminaries}

In this section, we recall some basic notations and facts following from $[3,8]$ that will appear along this paper.

Let $M^{n}$ be a connected, $n$-dimensional $(n \geq 2)$ oriented Riemannian manifold, $I \subseteq \mathbb{R}$ an interval, and $f: I \rightarrow \mathbb{R}$ a positive smooth function. We consider the product differential manifold $I \times M^{n}$ and denote by $\pi_{I}$ and $\pi_{M}$ the projections onto the base $I$ and fiber $M^{n}$, respectively. A particular class of semi-Riemannian manifolds is the one obtained by furnishing $I \times M^{n}$ with the metric

$$
\begin{aligned}
\langle v, w\rangle_{p}= & \epsilon\left\langle\left(\pi_{I}\right)_{*} v,\left(\pi_{I}\right)_{*} w\right\rangle \\
& +\left(f \circ \pi_{I}(p)\right)^{2}\left\langle\left(\pi_{M}\right)_{*} v,\left(\pi_{M}\right)_{*} w\right\rangle,
\end{aligned}
$$

for any $p \in \bar{M}^{n+1}$ and any $v, w \in T_{p} \bar{M}^{n+1}$, where $\epsilon= \pm 1$. We call such a space warped product manifold; $f$ is known as the warping function, and we denote the space by $\bar{M}^{n+1}=\epsilon I \times{ }_{f} M^{n}$. Note that $-I \times{ }_{f} M^{n}$ is called a generalized Robertson-Walker spacetime, in particular, $-I \times{ }_{f} M^{n}$ is called a Robertson-Walker spacetime if $M^{n}$ has constant sectional curvature. From [9], we know that a generalized Robertson-Walker spacetime has constant sectional curvature $\bar{k}$ if and only if the Riemannian fiber $M^{n}$ has constant sectional curvature $k$ and the warping function $f$ satisfies the following differential equation:

$$
\frac{f^{\prime \prime}}{f}=\bar{k}=\frac{f^{\prime 2}+k}{f^{2}} .
$$

It follows from [10] that the vector field $\left(f \circ \pi_{I}\right) \partial_{t}$ is conformal and closed (in this sense that its dual 1-form is closed) with conformal factor $\phi=f^{\prime} \circ \pi_{I}$, where the prime denotes differentiation with respect to $t \in I$. For $t_{0} \in I$, we orient the slice $\sum_{t_{0}}^{n}:=\left\{t_{0}\right\} \times M^{n}$ by using the unit normal vector field $\partial_{t}$, then from [11] we know $\Sigma_{t_{0}}^{n}$ has constant $r$ th mean curvature $H_{r}=-\epsilon\left(f^{\prime}\left(t_{0}\right) / f\left(t_{0}\right)\right)^{r}$ with respect to $\partial_{t}$.

A smooth immersion $\psi: \Sigma^{n} \rightarrow \epsilon I \times{ }_{f} M^{n}$ of an $n$-dimensional connected manifold $\Sigma^{n}$ is said to be a spacelike hypersurface if the induced metric via $\psi$ is a Riemannian metric on $\Sigma^{n}$. If $\Sigma^{n}$ is oriented by the unit vector field $N$, one obviously has $\epsilon=\epsilon_{\partial_{t}}=\epsilon_{N}$. Moreover, when $\epsilon=-1$, we may define the normal hyperbolic angle $\theta$ of $\Sigma^{n}$ as being the smooth function $\theta: \Sigma^{n} \rightarrow[0,+\infty)$ given by

$$
\cosh \theta=-\left\langle N, \partial_{t}\right\rangle
$$

We denote by $\bar{\nabla}$ and $\nabla$ the Levi-Civita connections in $\epsilon I \times{ }_{f} M^{n}$ and $\Sigma^{n}$, respectively. Then the Gauss-Weingarten formulas for the spacelike hypersurface $\psi: \Sigma^{n} \rightarrow \epsilon I \times_{f} M^{n}$ are given by

$$
\begin{gathered}
\bar{\nabla}_{X} Y=\nabla_{X} Y+\epsilon\langle A X, Y\rangle N \\
\bar{\nabla}_{X} N=-A X
\end{gathered}
$$

for any $X \in \Gamma\left(T \Sigma^{n}\right)$, where $A: \Gamma\left(T \Sigma^{n}\right) \rightarrow \Gamma\left(T \Sigma^{n}\right)$ be the shape operator of $\Sigma^{n}$ with respect to its Gauss map $N$, and $\Gamma\left(T \Sigma^{n}\right)$ denotes the Lie algebra of all tangential vector fields on $\Sigma^{n}$.

The curvature tensor $R$ of a spacelike hypersurface $\Sigma^{n}$ is given by [12] as the following:

$$
R(X, Y) Z=\nabla_{[X, Y]} Z-\left[\nabla_{X}, \nabla_{Y}\right] Z
$$

where [, ] denotes the Lie bracket and $X, Y, Z \in \Gamma\left(T \Sigma^{n}\right)$.

Let $\bar{R}$ and $R$ be the curvature tensors of $\epsilon I \times{ }_{f} M^{n}$ and $\Sigma^{n}$, respectively. Denote by $\bar{X}^{\top}$ the tangential component of a vector field $\bar{X} \in \Gamma\left(T \bar{M}^{n+1}\right)$; thus, for any $X, Y, Z \in \Gamma\left(T \Sigma^{n}\right)$ we have the following Gauss equation:

$R(X, Y) Z=(\bar{R}(X, Y) Z)^{\top}+\epsilon\langle A X, Z\rangle A Y-\epsilon\langle A Y, Z\rangle A X$

Consider a local orthonormal frame $\left\{E_{1}, \ldots, E_{n}\right\}$ and $X \in$ $\Gamma\left(T \Sigma^{n}\right)$, then the Ricci curvature tensor of $\Sigma^{n}$ is given as the following:

$$
\begin{gathered}
\operatorname{Ric}(X, X)=\sum_{i}\left\langle\bar{R}\left(X, E_{i}\right) X, E_{i}\right\rangle+n H\langle A X, X\rangle \\
-\epsilon\langle A X, A X\rangle .
\end{gathered}
$$

\section{Key Lemmas}

We consider two particular functions naturally attached to complete spacelike hypersurfaces, namely, the vertical (height) function $h=\left.\left(\pi_{I}\right)\right|_{\Sigma^{n}}$ and the support function 
$\left\langle N, \partial_{t}\right\rangle$. Denote by $\bar{\nabla}$ and $\nabla$ the gradients with respect to the metrics of $\epsilon I \times{ }_{f} M^{n}$ and $\Sigma^{n}$, respectively. Thus, by a simple computation, we have the gradient of $\pi_{I}$ on $\epsilon I \times{ }_{f} M^{n}$ as the following:

$$
\bar{\nabla} \pi_{I}=\epsilon\left\langle\bar{\nabla} \pi_{I}, \partial_{t}\right\rangle \partial_{t}=\epsilon \partial_{t}
$$

Thus, the gradient of $h$ on $\Sigma^{n}$ is given by

$$
\nabla h=\left(\bar{\nabla} \pi_{I}\right)^{\top}=\epsilon\left(\partial_{t}\right)^{\top}=\epsilon \partial_{t}-\left\langle N, \partial_{t}\right\rangle N
$$

We denote by $|\cdot|$ the norm of a vector field on $\Sigma^{n}$, then we get

$$
|\nabla h|^{2}=\epsilon\left(1-\left\langle N, \partial_{t}\right\rangle^{2}\right)
$$

According to [4], a spacelike hypersurface $\psi: \sum^{n} \rightarrow$ $-I \times_{f} M^{n}$ is said to be bounded away from the future infinity of $-I \times{ }_{f} M^{n}$ if there exists $\bar{t} \in I$ such that

$$
\psi\left(\Sigma^{n}\right) \subset\left\{(t, p) \in-I \times_{f} M^{n}: t \leq \bar{t}\right\}
$$

Analogously, a spacelike hypersurface $\psi: \Sigma^{n} \rightarrow-I \times_{f} M^{n}$ is said to be bounded away from the past infinity of $-I \times{ }_{f} M^{n}$ if there exists $\underline{t} \in I$ such that

$$
\psi\left(\Sigma^{n}\right) \subset\left\{(t, p) \in-I \times_{f} M^{n}: t \geq \underline{t}\right\}
$$

Finally, $\Sigma^{n}$ is said to be bounded away from the infinity of $-I \times_{f} M^{n}$ if it is both bounded away from the past and future infinity of $-I \times{ }_{f} M^{n}$.

In order to prove our main theorems, we will make use of the following computations. We also refer the reader to $[6,11]$ for a more generalized proposition which makes the following lemma as its trivial case.

Lemma 3. Let $\psi: \Sigma^{n} \rightarrow \epsilon I \times{ }_{f} M^{n}$ be a spacelike hypersurface immersed in a semi-Riemannian warped product. If

$$
\sigma(t)=\int_{t_{0}}^{t} f(s) d s
$$

then

$$
\Delta \sigma(h)=\epsilon n\left(f^{\prime}(h)+f(h)\left\langle N, \partial_{t}\right\rangle H\right)
$$

where $\Delta$ denotes the Laplacian operator and $h$ is the height function of $\Sigma^{n}$.

Proof. Let $\nabla$ be the gradient with respect to the metric of $\Sigma^{n}$ induced from $\bar{M}^{n+1}$. Thus, it is easy to see

$$
\nabla \sigma(h)=f(h) \nabla h .
$$

Denote by $\left\{e_{1}, \ldots, e_{n}\right\}$ a local orthonormal frame of $\Gamma\left(T \Sigma^{n}\right)$. By the definition of Laplacian operator and using (22), then we have

$$
\begin{aligned}
\Delta \sigma(h) & :=\operatorname{div}(\nabla \sigma(h))=\operatorname{div}(f(h) \nabla h) \\
& =\sum_{i}\left\langle e_{i}, \nabla_{e_{i}}(f(h) \nabla h)\right\rangle \\
& =\sum_{i}\left\langle e_{i}, f(h) \nabla_{e_{i}} \nabla h\right\rangle+\sum_{i}\left\langle e_{i}, e_{i}(f(h)) \nabla h\right\rangle \\
& =f(h) \sum_{i}\left\langle e_{i}, \nabla_{e_{i}} \nabla h\right\rangle+\sum_{i} e_{i}(f(h))\left\langle e_{i}, \nabla h\right\rangle \\
& =f(h) \Delta h+f^{\prime}(h) \sum_{i}\left\langle e_{i}, \nabla h\right\rangle\left\langle e_{i}, \nabla h\right\rangle \\
& =f(h) \Delta h+f^{\prime}(h)|\nabla h|^{2} .
\end{aligned}
$$

By taking $r=1$ in Lemma 2.2 of [13] (we also refer the reader to Lemma 4.1 of [11]), then we have

$$
\Delta h=(\ln f)^{\prime}(h)\left(\epsilon n-|\nabla h|^{2}\right)+\epsilon n H\left\langle N, \partial_{t}\right\rangle .
$$

By substituting (24) into (23), we complete the proof.

We need another lemma proved by Albujer et al. in [3].

Lemma 4. Let $\psi: \Sigma^{n} \rightarrow-I \times{ }_{f} M^{n}$ be a spacelike hypersurface immersed in a Robertson-Walker spacetime whose Riemannian fiber $M^{n}$ has constant sectional curvature $k$. Denote by $h$ the height function on $\Sigma^{n}$. If $-I \times{ }_{f} M^{n}$ obeys the null convergence condition, then

$$
\sum_{i}\left\langle\bar{R}\left(X, E_{i}\right) X, E_{i}\right\rangle \geq(n-1) \frac{k+f^{\prime 2}(h)}{f^{2}(h)}|X|^{2},
$$

where $\bar{R}$ is the Riemannian curvature tensor of $-I \times{ }_{f} M^{n}$ and $X \in \Gamma\left(T \Sigma^{n}\right)$.

Remark 5. It follows from (14) and Lemma 4 that, if the Riemannian fiber has constant sectional curvature, the Ricci curvature tensor of $\Sigma^{n}$ is bounded from below if $\Sigma^{n}$ is bounded away from the infinity of $\bar{M}^{n+1}$.

We also need another lemma shown by Aquino and de Lima in [5].

Lemma 6. Let $\bar{M}^{n+1}=I \times_{f} M^{n}$ be a warped product which satisfies convergence condition (5). Let $\psi: \sum^{n} \rightarrow \bar{M}^{n+1}$ be a complete hypersurface with both mean curvature $H$ and second fundamental form A bounded. If $f^{\prime \prime} / f$ is bounded on $\Sigma^{n}$, then the Ricci curvature of $\Sigma^{n}$ is bounded from below.

In order to prove our main theorems, we also need the well-known generalized maximal principal due to Omori [1] and Yau [2].

Lemma 7. Let $\Sigma^{n}$ denote an $n$-dimensional complete Riemannian manifold whose Ricci curvature tensor is bounded from 
below. Then, for any $\mathscr{C}^{2}$-function $u: \Sigma^{n} \rightarrow \mathbb{R}$ with $u^{*}=$ $\sup _{\Sigma^{n}} u<\infty$, there exists a sequence of points $\left\{p_{k}\right\}_{k \in N}$ in $\Sigma^{n}$ satisfying the following properties:

$$
\begin{aligned}
& \text { (i) } \lim _{k \rightarrow \infty} u\left(p_{k}\right)=\sup _{\Sigma^{n}} u, \quad \text { (ii) } \lim _{k \rightarrow \infty}|\nabla u|\left(p_{k}\right)=0, \\
& \text { (iii) } \lim _{k \rightarrow \infty} \Delta u\left(p_{k}\right) \leq 0 \text {. }
\end{aligned}
$$

Equivalently, for any $\mathscr{C}^{2}$-function $u: \Sigma^{n} \rightarrow \mathbb{R}$ with $u_{*}=$ $\inf _{\Sigma^{n}} u>-\infty$, there exists a sequence of points $\left\{q_{k}\right\}_{k \in N}$ in $\Sigma^{n}$ satisfying the following properties:

$$
\begin{gathered}
\text { (i) } \lim _{k \rightarrow \infty} u\left(q_{k}\right)=\inf _{\Sigma^{n}} u, \quad \text { (ii) } \lim _{k \rightarrow \infty}|\nabla u|\left(q_{k}\right)=0, \\
\text { (iii) } \lim _{k \rightarrow \infty} \Delta u\left(q_{k}\right) \geq 0 .
\end{gathered}
$$

\section{Proofs of Main Theorems}

Proof of Theorem 1. Since $\partial_{t}$ is a unitary timelike vector field globally defined on the ambient spacetime, then there exists a unique timelike unitary normal vector filed $N$ globally defined on the spacelike hypersurface $\Sigma^{n}$ which is the same time orientation as $\partial_{t}$. By using the reverse Cauchy-Schwarz inequality, we have

$$
\left.\left\langle N, \partial_{t}\right\rangle\right|_{\Sigma^{n}} \leq-1<0 .
$$

We observe that, from Lemma 3 ,

$$
\Delta \sigma(h)=-n\left(f^{\prime}(h)+f(h)\left\langle N, \partial_{t}\right\rangle H\right) .
$$

By using the assumption (3), we have that $H \leq 0$. Then, it follows from (28) that $\left\langle N, \partial_{t}\right\rangle H \geq-H \geq 0$. Notice that the warping function is positive on $I$, then from (3) and (29), we have the following inequality:

$$
\begin{aligned}
\Delta \sigma(h) & =-n f(h)\left(\frac{f^{\prime}(h)}{f(h)}+\left\langle N, \partial_{t}\right\rangle H\right) \\
& \leq-n f(h)\left(\frac{f^{\prime}(h)}{f(h)}-H\right) \\
& \leq-n f(h)\left(\inf _{\Sigma^{n}} \frac{f^{\prime}(h)}{f(h)}-H\right) \\
& \leq 0 .
\end{aligned}
$$

On the other hand, since the spacelike hypersurface is bounded away from the infinity of $\bar{M}^{n+1}$, and the constant sectional curvature of the fiber satisfies the null convergence condition, then it follows from Lemma 4 and Remark 5 that the Ricci curvature tensor of $\Sigma^{n}$ is bounded from below. Then by the definition of $\sigma(h)$, that is, (20), it is easy to see $\inf _{\Sigma^{n}} \sigma(h)<\infty$. Thus, applying Lemma 7 to the smooth function $\sigma(h)$ on $\Sigma^{n}$ implies that there exists a sequence of points $\left\{q_{k}\right\}_{k \in N}$ in $\Sigma^{n}$ with the following properties:

$$
\begin{gathered}
\operatorname{Lim}_{k \rightarrow \infty} \sigma(h)\left(q_{k}\right)=\inf _{\Sigma^{n}} \sigma(h)>-\infty, \\
\lim _{k \rightarrow \infty} \Delta \sigma(h)\left(q_{k}\right) \geq 0 .
\end{gathered}
$$

Note that $H$ is a nonpositive constant, then it follows from (30) that

$$
\lim _{k \rightarrow \infty} \Delta \sigma(h)\left(q_{k}\right) \leq-n \lim _{k \rightarrow \infty} f(h)\left(q_{k}\right)\left(\inf _{\Sigma^{n}} \frac{f^{\prime}(h)}{f(h)}-H\right) \leq 0 .
$$

Since the warping function is positive on $\Sigma^{n}$ and $\Sigma^{n}$ is bounded away from the infinity, from (31) and (32), we have $\lim _{k \rightarrow \infty} \Delta \sigma(h)\left(q_{k}\right)=0$, which means that

$$
\inf _{\Sigma^{n}} \frac{f^{\prime}(h)}{f(h)}-H=0 .
$$

Substituting the above equation into (4) implies that $|\nabla h|^{2} \equiv 0$, that is, $h$ is a constant on $\Sigma^{n}$; thus $\Sigma^{n}$ is a slice of $\bar{M}^{n+1}$.

From (9), we see that every Robertson-Walker spacetime with constant sectional curvature trivially obeys the null convergence condition (2). Therefore, from Theorem 1, we obtain the following result.

Corollary 8. Let $\bar{M}^{n+1}=-I \times{ }_{f} M^{n}$ be a Robertson-Walker spacetime with constant sectional curvature. Let $\psi: \Sigma^{n} \rightarrow$ $\bar{M}^{n+1}$ be a complete spacelike hypersurface with constant mean curvature $H$. Suppose that $\Sigma^{n}$ is bounded away from the infinity. If (3) and (4) hold, then $\Sigma^{n}$ is a slice.

Theorem 9. Let $\bar{M}^{n+1}=-I \times{ }_{f} M^{n}$ be a Robertson-Walker spacetime whose Riemannian fiber $M^{n}$ has constant sectional curvature $k$ satisfying the null convergence condition. Let $\psi: \Sigma^{n} \rightarrow \bar{M}^{n+1}$ be a complete spacelike hypersurface with constant mean curvature $H$ and bounded away from the infinity. If

$$
H \geq \max \left\{\sup _{p \in \Sigma^{n}} \frac{f^{\prime}}{f}(h(p)), 0\right\},
$$

and the height function $h$ of $\Sigma^{n}$ satisfies

$$
|\nabla h|^{2} \leq \alpha\left|H-\sup _{p \in \Sigma^{n}} \frac{f^{\prime}}{f}(h(p))\right|^{\beta},
$$

for some positive constant $\alpha$ and $\beta$, then $\Sigma^{n}$ is a slice.

Proof. From (34), we see that the constant mean curvature $H$ is nonnegative, then it follows from (28) and (34) that $\left\langle N, \partial_{t}\right\rangle H \leq-H \leq 0$. Thus, from (29), we have

$$
\begin{aligned}
\Delta \sigma(h) & =-n f(h)\left(\frac{f^{\prime}(h)}{f(h)}+\left\langle N, \partial_{t}\right\rangle H\right) \\
& \geq-n f(h)\left(\frac{f^{\prime}(h)}{f(h)}-H\right) \\
& \geq-n f(h)\left(\sup _{\Sigma^{n}} \frac{f^{\prime}(h)}{f(h)}-H\right) \\
& \geq 0 .
\end{aligned}
$$


Using the similar analysis with the proof of Theorem 1, applying the Lemma 7 to the smooth function $\sigma(h)$ on $\Sigma^{n}$ implies that there exists a sequences of points $\left\{p_{k}\right\}_{k \in N}$ in $\Sigma^{n}$ satisfying the following properties:

$$
\begin{gathered}
\operatorname{Lim}_{k \rightarrow \infty} \sigma(h)\left(p_{k}\right)=\sup _{\Sigma^{n}} \sigma(h)<\infty, \\
\lim _{k \rightarrow \infty} \Delta \sigma(h)\left(p_{k}\right) \leq 0 .
\end{gathered}
$$

It follows from (36) that

$$
\lim _{k \rightarrow \infty} \Delta \sigma(h)\left(p_{k}\right) \geq-n \lim _{k \rightarrow \infty} f(h)\left(p_{k}\right)\left(\sup _{\Sigma^{n}} \frac{f^{\prime}(h)}{f(h)}-H\right) \geq 0 .
$$

From (37) and (38), we have $\lim _{k \rightarrow \infty} \Delta \sigma(h)\left(p_{k}\right)=0$. Thus, from (36), we get

$$
\sup _{\Sigma^{n}} \frac{f^{\prime}(h)}{f(h)}-H=0 .
$$

By substituting the above equation into (35), we have $|\nabla h|^{2} \equiv 0$, that is, $h$ is a constant on $\Sigma^{n}$; thus $\Sigma^{n}$ is a slice.

Corollary 10. Let $\bar{M}^{n+1}=-I \times{ }_{f} M^{n}$ be a Robertson-Walker spacetime with constant sectional curvature. Let $\psi: \Sigma^{n} \rightarrow$ $\bar{M}^{n+1}$ be a complete spacelike hypersurface with constant mean curvature $H$. Suppose that $\Sigma^{n}$ is bounded away from the infinity. If (34) and (35) hold, then $\Sigma^{n}$ is a slice.

Noting that a Robertson-Walker spacetime with constant sectional curvature trivially obeys the null convergence condition; thus the proof of Corollary 10 follows from Theorem 9.

It is well known that Robertson-Walker spacetime is called static Robertson-Walker spacetime if the warping function $f$ is constant. Without losing the generality, we consider $f=1$ in the following. In this case, the null convergence condition (2) implies that $k \geq 0$. Also, (3) becomes that $H \leq 0$. When $f$ is a constant, (33) (or (39)) implies that $H=0$, that is, $\Sigma^{n}$ is maximal. On the other hand, letting the warping function $f$ be a constant, we see from (34) that $H \geq$ 0 . Then a weaker assumption than (4) and (35), that is, the normal hyperbolic angle is bounded on $\Sigma^{n}$, also guarantees Theorems 1 and 9. Thus, from Theorems 1 and 9 , we have the following corollary.

Corollary 11. Let $\bar{M}^{n+1}=-I \times M^{n}$ be a static RobertsonWalker spacetime whose Riemannian fiber $M^{n}$ has nonnegative constant sectional curvature $k$. Let $\psi: \Sigma^{n} \rightarrow \bar{M}^{n+1}$ be a complete, connected spacelike hypersurface with constant mean curvature $H$. Suppose that the normal hyperbolic angle of $\Sigma^{n}$ is bounded, then $\Sigma^{n}$ is a maximal slice.

We also refer the reader to $[3,14]$ for the similar results with Corollary 11 proved by using the different methods with ours. Next, we give the proof of Theorem 2, which is the Riemannian warped product version of our Theorem 1.
Proof of Theorem 2. Initially, we consider that the orientation $N$ of the hypersurface such that its angle function satisfies

$$
-1 \leq\left\langle N, \partial_{t}\right\rangle \leq 0 .
$$

Then from Lemma 3, we have that

$$
\begin{aligned}
\Delta \sigma(h) & =n\left(f^{\prime}(h)+f(h)\left\langle N, \partial_{t}\right\rangle H\right) \\
& =n f(h)\left(\frac{f^{\prime}(h)}{f(h)}+\left\langle N, \partial_{t}\right\rangle H\right) .
\end{aligned}
$$

By using the assumption (6) we see that the mean curvature $H$ is non-positive, then it follows from (40) that $0 \leq H\left\langle N, \partial_{t}\right\rangle \leq-H$. Notice that the warping function $f$ is positive on $I$, then from (41) we have the following inequality:

$$
\begin{aligned}
\Delta \sigma(h) & =n f(h)\left(\frac{f^{\prime}(h)}{f(h)}+\left\langle N, \partial_{t}\right\rangle H\right) \\
& \leq n f(h)\left(\frac{f^{\prime}(h)}{f(h)}-H\right) \\
& \leq n f(h)\left(\sup _{\Sigma^{n}} \frac{f^{\prime}(h)}{f(h)}-H\right) \\
& \leq 0 .
\end{aligned}
$$

Since the spacelike hypersurface is bounded away from the infinity, it follows that $f^{\prime \prime} / f$ is bounded on $\Sigma^{n}$. Let $S_{2}$ denote the second elementary symmetric function on the eigenvalues of the shape operator $A$, and $H_{2}=(2 / n(n-1)) S_{2}$ denotes the mean value of $S_{2}$. It is easy to see $|A|^{2}=n^{2} H^{2}-$ $n(n-1) H_{2}$. Then the assumption that $H_{2}$ is bounded from below means that $A$ is bounded from above; from Lemma 6 we know that the Ricci curvature tensor of $\Sigma^{n}$ is bounded from below.

Finally, by applying analogous arguments employed in the last part of the proof of Theorem 1, we conclude that $\Sigma^{n}$ is a slice of $I \times_{f} M^{n}$.

\section{Application of Main Theorems}

In this section, we consider a particular model of Lorentzian warped product, namely, the steady state space, that is, the warped product

$$
\mathscr{H}^{n+1}=-\mathbb{R} \times{ }_{e^{t}} \mathbb{R}^{n} .
$$

The importance of considering $\mathscr{H}^{n+1}$ comes from the fact that, in cosmology, $\mathscr{H}^{4}$ is the steady model of the universe proposed by Bondi and Gold [15] and Hoyle [16]. Moreover, in physical context the steady state space appears naturally as an exact solution for the Einstein equations, being a cosmological model where matter is supposed to travel along geodesic normal to horizontal hyperplanes. We also notice that Montiel [17] gave an alternative description of the steady state space $\mathscr{H}^{n+1}$ as follows. 
Let $\mathbb{L}_{1}^{n+2}$ denote the $(n+2)$-dimensional LorentzianMinkowski space, that is, the real vector space $\mathbb{R}^{n+2}$ with a Lorentzian metric

$$
\langle v, w\rangle=\sum_{i=1}^{n+1} v_{i} w_{i}-v_{n+2} w_{n+2},
$$

for all $v, w \in \mathbb{R}^{n+2}$. The $(n+1)$-dimensional de Sitter space $\mathbb{S}_{1}^{n+1}$ is defined as the following: $\mathbb{S}_{1}^{n+1}=\left\{p \in \mathbb{L}_{1}^{n+2}:\langle p, p\rangle=\right.$ 1 . Let $q \in \mathbb{L}_{1}^{n+2}$ be a nonzero null vector of the null cone with vertex in the origin, such that $\left\langle q, e_{n+2}\right\rangle>0$, where $e_{n+2}=$ $(0, \ldots, 0,1)$. Then, it can be shown that the open region

$$
\left\{p \in \mathbb{S}_{1}^{n+1}:\langle p, q\rangle>0\right\}
$$

of the de Sitter space $\mathbb{S}_{1}^{n+1}$ is isometric to $\mathscr{H}^{n+1}$ (see [18] for details).

Recently, some uniqueness theorems for steady state space were obtained by $[13,19]$. In fact, Caminha and de Lima [19] proved the following results.

Theorem 12. Let $\psi: \Sigma^{2} \rightarrow \mathscr{H}^{3}$ be a Riemannian immersion of a complete surface of non-negative Guassian curvature $K_{\Sigma^{n}}$ with constant mean curvature $H \geq 1$. If

$$
|\nabla h|^{2} \leq H^{2}-1
$$

then $\psi\left(\Sigma^{n}\right)$ is a slice of $\mathscr{H}^{3}$.

Suppose that the warping function $f(t)=e^{t}$; thus (34) becomes $H \geq 1$; meanwhile the inequality (35) becomes

$$
|\nabla h|^{2} \leq \alpha|H-1|^{\beta}
$$

where both $\alpha$ and $\beta$ are positive constants. Suppose that $\beta=$ 2, then we have $\alpha(H-1)^{\beta} \geq H^{2}-1$ for all $H \geq 1$ if $\alpha \geq$ $1+2 /(H-1)>0$. Thus, our Theorem 9 extends Theorem 4.5 in [19]. At last, we write the uniqueness theorems for surface in steady state space which follows from Theorems 1 and 9, respectively, as following.

Theorem 13. Let $\psi: \Sigma^{2} \rightarrow \mathscr{H}^{3}$ be a Riemannian immersion of a complete surface with constant mean curvature $H \leq 0$. If

$$
|\nabla h|^{2} \leq \alpha(1-H)^{\beta}
$$

holds for some positive constant $\alpha$ and $\beta$, then $\psi\left(\Sigma^{n}\right)$ is a slice of $\mathscr{H}^{3}$.

Theorem 14. Let $\psi: \Sigma^{2} \rightarrow \mathscr{H}^{3}$ be a Riemannian immersion of a complete surface with constant mean curvature $H \geq 1$. If

$$
|\nabla h|^{2} \leq \alpha(H-1)^{\beta}
$$

holds for some positive constant $\alpha$ and $\beta$, then $\psi\left(\sum^{n}\right)$ is a slice of $\mathscr{H}^{3}$.

\section{Entire Vertical Graphs in Robertson-Walker Spacetime}

In the last section of this paper, we investigate the applications of our main theorems for entire vertical graphs in a Robertson-Walker spacetime. We follow [3, 8, 20] for the basic notations and facts used in this section.

Let $\Omega \subseteq M^{n}$ be a connected domain of $M^{n}$; a vertical graph over $\Omega$ is defined by smooth function $u \in \mathscr{C}^{\infty}(\Omega)$ and it is given by

$$
\Sigma^{n}(u)=\left\{(u(p), p): p \in \Omega \subseteq M^{n}\right\} \subset-I \times_{f} M^{n} .
$$

The metric induced on $\Omega$ from the Lorentzian metric on the ambient space via $\Sigma^{n}$ is $\langle\rangle=,-d u^{2}+f^{2}(u)\langle,\rangle_{M^{n}}$. The graph is said to be entire if $\Omega=M^{n}$. It is easy to see that a graph $\Sigma(u)$ is a spacelike hypersurface if and only if $|D u|_{M^{n}}^{2}<f^{2}(u)$, where $D u$ is the gradient of $u$ in $\Omega$ and $|D u|_{M^{n}}$ its norm, both with respect to the metric $\langle,\rangle_{M^{n}}$ in $\Omega$.

Let $\Sigma^{n}(u)$ be a spacelike vertical graph over a domain $\Omega$, its future-pointing Gauss map is given by the vector field

$$
\begin{aligned}
N(p)= & \frac{f(u(p))}{\sqrt{f^{2}(u(p))-|D u(p)|_{M^{n}}^{2}}} \\
& \times\left(\left.\partial_{t}\right|_{(u(p), p)}+\frac{1}{f^{2}(u(p))} D u(p)\right), \quad p \in \Omega .
\end{aligned}
$$

Moreover, the shape operator $A$ of $\Sigma^{n}(u)$ with respect to $N$ is given by

$$
\begin{aligned}
A X= & -\frac{1}{f(u) \sqrt{f^{2}(u)-|D u|_{M^{n}}^{2}}} D_{X} D u \\
& -\frac{f^{\prime}(u)}{\sqrt{f^{2}(u)-|D u|_{M^{n}}^{2}}} X \\
& +\left(\frac{f^{\prime}(u)\langle D u, X\rangle}{\left(f^{2}(u)-|D u|_{M^{n}}^{2}\right)^{3 / 2}}\right. \\
& \left.-\frac{\left\langle D_{X} D u, D u\right\rangle_{M^{n}}}{\left.f(u)\left(f^{2}(u)-|D u|_{M^{n}}^{2}\right)\right)^{3 / 2}}\right) D u,
\end{aligned}
$$

for any tangent vector field $X$ on $\Omega$, where $D$ denotes the Levi-Civita connection in $\Omega$ with respect to the metric $\langle,\rangle_{M^{n}}$. The mean curvature function of a spacelike graph $\Sigma^{n}(u)$ with respect to $N$ is given as the following:

$$
\begin{aligned}
& \operatorname{Div}\left(\frac{D u}{\sqrt{f^{2}(u)-|D u|_{M^{n}}^{2}}}\right) \\
& \quad=n f(u)\left(H(u)-\frac{f^{\prime}(u)}{\sqrt{f^{2}(u)-|D u|_{M^{n}}^{2}}}\right),
\end{aligned}
$$


where Div denotes the divergence operator on $\Omega$ with respect to the metric $\langle,\rangle_{M^{n}}$. Now, we give the following nonparametric version of Theorem 1.

Theorem 15. Let $\bar{M}^{n+1}=-I \times{ }_{f} M^{n}$ be a Robertson-Walker spacetime whose Riemannian fiber $M^{n}$ has constant sectional curvature $k$. Suppose that $\bar{M}^{n+1}$ obeys the null convergence condition (2). Let $\Sigma^{n}(u)$ be an entire spacelike vertical graph in $\bar{M}^{n+1}$ with constant mean curvature $H$ and bounded away from the infinity. If

$$
\begin{gathered}
H \leq \min \left\{\inf _{p \in \Sigma^{n}} \frac{f^{\prime}}{f}(u(p)), 0\right\}, \\
|D u|_{M^{n}}^{2} \leq \alpha \inf _{\Sigma^{n}(u)} f^{2}(u) \frac{\left|\inf _{\Sigma^{n}(u)}\left(f^{\prime} / f\right)(u)-H\right|^{\beta}}{1+\alpha\left|\inf _{\Sigma^{n}(u)}\left(f^{\prime} / f\right)(u)-H\right|^{\beta}},
\end{gathered}
$$

for some positive constant $\alpha$ and $\beta$, then $\Sigma^{n}(u)$ is a slice.

Proof. First of all and following the ideas of Albujer et al. in the proof of Theorem 4.1 in [20] and Theorem 4.1 in [3], it can be easily seen that the induced metric on the entire graph $\Sigma^{n}(u)$ is complete. Then it follows from $[8,20]$ that

$$
|\nabla h|^{2}=\frac{|D u|_{M^{n}}^{2}}{f^{2}(u)-|D u|_{M^{n}}^{2}} .
$$

Thus, together with (55) and (56) and by a straightforward computation, we know that (4) holds. Then the proof of Theorem 15 follows from Theorem 1 .

Let $\bar{M}^{n+1}=-I \times{ }_{f} M^{n}$ be a static Robertson-Walker spacetime, that is, $f=1$. Thus, the null convergence condition implies that $k \geq 0$ and (54) becomes $H \leq 0$. In particular, from (55), we get

$$
|D u|_{M^{n}}^{2} \leq \alpha \frac{|H|^{\beta}}{1+\alpha|H|^{\beta}} .
$$

Following from Theorem 15, we get a uniqueness theorem for entire spacelike vertical graphs in static spacetime.

Theorem 16. Let $\bar{M}^{n+1}=-I \times M^{n}$ be a static RobertsonWalker spacetime whose Riemannian fiber $M^{n}$ has nonnegative constant sectional curvature $k$. Let $\Sigma^{n}(u)$ be an entire spacelike vertical graph in $\bar{M}^{n+1}$ with non-positive constant mean curvature $H$ and bounded away from the infinity. If the condition (57) is satisfied for some positive constant $\alpha$ and $\beta$, then $\Sigma^{n}(u)$ is a slice.

\section{Acknowledgments}

The project is supported by Natural Science Foundation of China (no. 10931005) and Natural Science Foundation of Guangdong Province of China (no. S2011010000471).

\section{References}

[1] H. Omori, "Isometric immersions of Riemannian manifolds," Journal of the Mathematical Society of Japan, vol. 19, pp. 205214, 1967.

[2] S. T. Yau, "Harmonic functions on complete Riemannian manifolds," Communications on Pure and Applied Mathematics, vol. 28, pp. 201-228, 1975.

[3] A. L. Albujer, F. E. C. Camargo, and H. F. de Lima, "Complete spacelike hypersurfaces in a Robertson-Walker spacetime," Mathematical Proceedings of the Cambridge Philosophical Society, vol. 151, no. 2, pp. 271-282, 2011.

[4] K. Akutagawa, "On spacelike hypersurfaces with constant mean curvature in the de Sitter space," Mathematische Zeitschrift, vol. 196, no. 1, pp. 13-19, 1987.

[5] C. P. Aquino and H. F. de Lima, "On the rigidity of constant mean curvature complete vertical graphs in warped products," Differential Geometry and its Applications, vol. 29, no. 4, pp. 590-596, 2011.

[6] L. J. Alías, D. Impera, and M. Rigoli, "Spacelike hypersurfaces of constant higher order mean curvature in generalized Robertson-Walker spacetimes," Mathematical Proceedings of the Cambridge Philosophical Society, vol. 152, no. 2, pp. 365-383, 2012.

[7] H. F. de Lima and J. R. de Lima, "Complete hypersurfaces immersed in a semi-Riemannian warped product," Differential Geometry and Its Applications, vol. 30, no. 1, pp. 136-143, 2012.

[8] L. J. Alías, A. Romero, and M. Sánchez, "Uniqueness of complete spacelike hypersurfaces of constant mean curvature in generalized Robertson-Walker spacetimes," General Relativity and Gravitation, vol. 27, no. 1, pp. 71-84, 1995.

[9] L. J. Alías, A. Romero, and M. Sánchez, "Spacelike hypersurfaces of constant mean curvature and Calabi-Bernstein type problems," The Tohoku Mathematical Journal, vol. 49, no. 3, pp. 337345, 1997.

[10] S. Montiel, "Unicity of constant mean curvature hypersurfaces in some Riemannian manifolds," Indiana University Mathematics Journal, vol. 48, no. 2, pp. 711-748, 1999.

[11] L. J. Alías and A. G. Colares, "Uniqueness of spacelike hypersurfaces with constant higher order mean curvature in generalized Robertson-Walker spacetimes," Mathematical Proceedings of the Cambridge Philosophical Society, vol. 143, no. 3, pp. 703-729, 2007.

[12] B. O'Neill, Semi-Riemannian Geometry with Applications to Relativity, vol. 103 of Pure and Applied Mathematics, Academic Press, New York, NY, USA, 1983.

[13] F. Camargo, A. Caminha, and H. de Lima, "Bernstein-type theorems in semi-Riemannian warped products," Proceedings of the American Mathematical Society, vol.139, no. 5, pp.1841-1850, 2011.

[14] G. Li and I. Salavessa, "Graphic Bernstein results in curved pseudo-Riemannian manifolds," Journal of Geometry and Physics, vol. 59, no. 9, pp. 1306-1313, 2009.

[15] H. Bondi and T. Gold, "On the generation of magnetism by fluid motion," Monthly Notices of the Royal Astronomical Society, vol. 110, pp. 607-611, 1950.

[16] F. Hoyle, "A new model for the expanding universe," Monthly Notices of the Royal Astronomical Society, vol. 108, pp. 372-382, 1948.

[17] S. Montiel, "Complete non-compact spacelike hypersurfaces of constant mean curvature in de Sitter spaces," Journal of the Mathematical Society of Japan, vol. 55, no. 4, pp. 915-938, 2003. 
[18] A. L. Albujer and L. J. Alías, "Spacelike hypersurfaces with constant mean curvature in the steady state space," Proceedings of the American Mathematical Society, vol. 137, no. 2, pp. 711-721, 2009.

[19] A. Caminha and H. F. de Lima, "Complete vertical graphs with constant mean curvature in semi-Riemannian warped products," Bulletin of the Belgian Mathematical Society: Simon Stevin, vol. 16, no. 1, pp. 91-105, 2009.

[20] A. L. Albujer, F. E. C. Camargo, and H. F. de Lima, "Complete spacelike hypersurfaces with constant mean curvature in $-\mathbb{R} \times$ $\mathbb{T}^{n}$,' Journal of Mathematical Analysis and Applications, vol. 368, no. 2, pp. 650-657, 2010. 


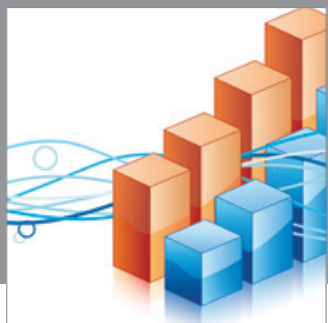

Advances in

Operations Research

mansans

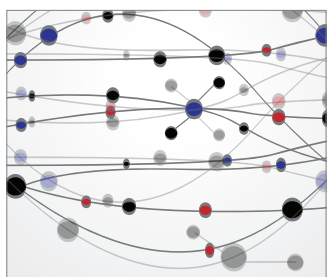

The Scientific World Journal
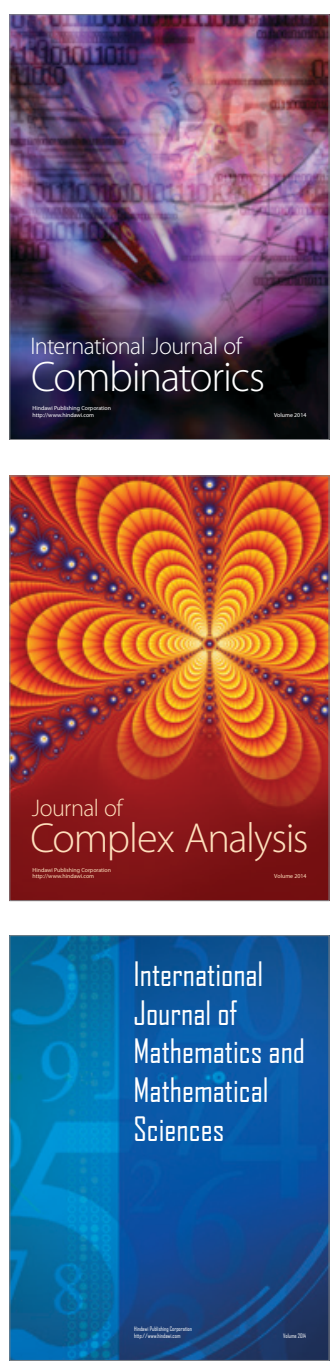
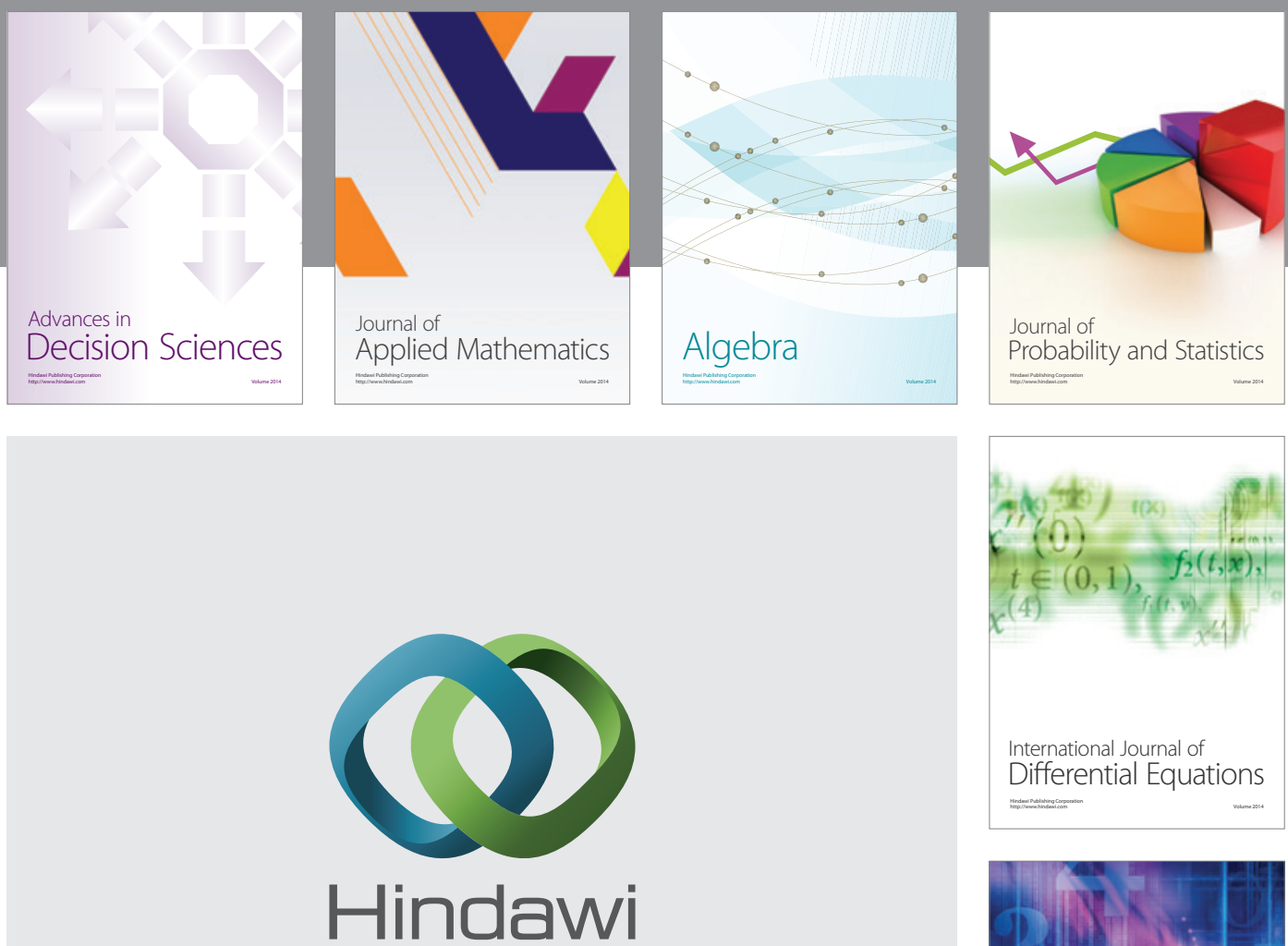

Submit your manuscripts at http://www.hindawi.com
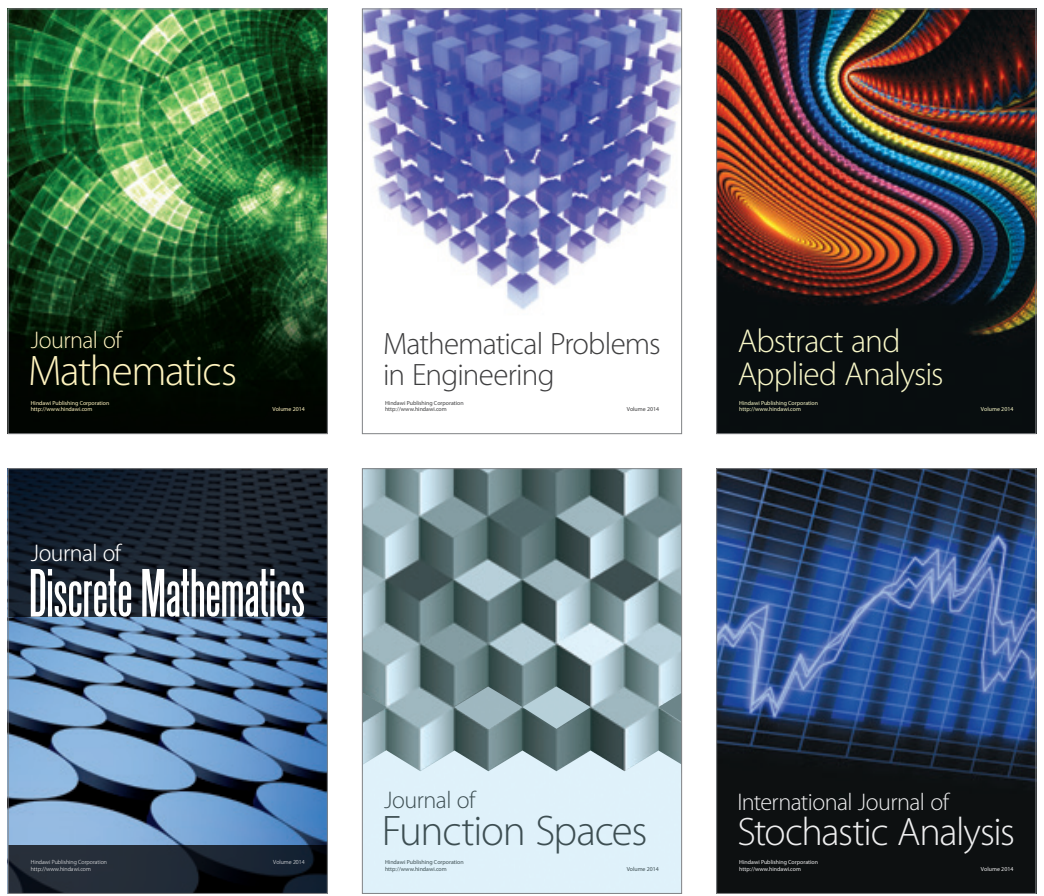

Journal of

Function Spaces

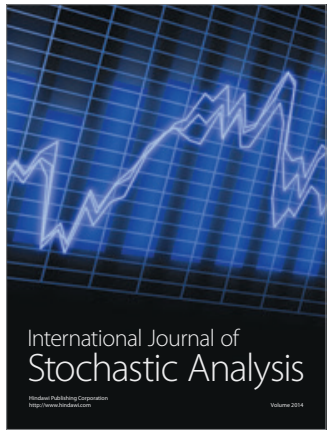

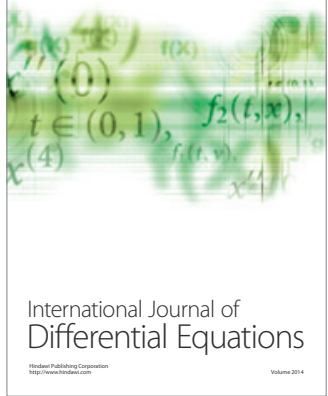
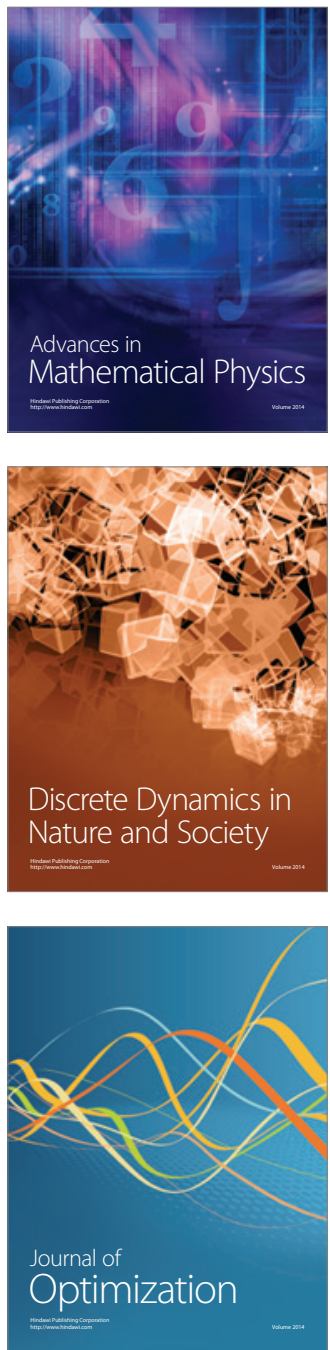